\title{
CIRBP is a novel oncogene in human bladder cancer inducing expression of HIF-1a
}

\author{
Mengxin Lu', Qiangqiang Ge', Gang Wang ${ }^{2,3,4}$, Yongwen Luo ${ }^{1}$, Xiaolong Wang ${ }^{5}$, Wei Jiang ${ }^{2,3,6}$, Xuefeng Liu (1) \\ Chin-Lee $\mathrm{Wu}^{8}$, Yu Xiao ${ }^{1,2,3,4}$ and Xinghuan Wang ${ }^{1,3}$
}

\begin{abstract}
Cold-inducible RNA binding protein (CIRBP) has been reported to be associated with distinct tumorigenesis. In this study, we investigated the role of CIRBP in human bladder cancer (BCa), indicating that CIRBP is overexpressed in BCa tissues and cell lines to promote proliferation and migration. Moreover, CIRBP could induce expression of HIF-1a via binding to the $3^{\prime}$-UTR of its mRNA to increase the mRNA stability in BCa cells. Furthermore, we demonstrated that PTGIS is a HIF-1 a targeted gene, a major regulator in hypoxic cancer progression by activating transcription of various oncogenes. Our results also suggested that overexpression of HIF-1a may suppress the expression of PTGIS in BCa cells, by binding to HRE sequence at the promoter region of PTGIS. In addition, we found a strongly downregulation of PTGIS in BCa tissue and transcriptionally inhibited by HIF-1a in BCa cells, which could be triggered by its DNA methylation. Further result suggested that knockdown of CIRBP could promote the expression of PTGIS, meanwhile knockdown of PTGIS could partially rescue CIRBP-deficiency induced inhibition of migration and proliferation in BCa cells. Taken together, our study indicated that CIRBP could be a novel oncogene in human bladder cancer inducing transcription of HIF-1a, which could inhibit expression of methylated PTGIS.
\end{abstract}

\section{Introduction}

Bladder cancer $(\mathrm{BCa})$ is the ninth most common malignancy worldwide, with an estimated 429,000 new cases and 165,000 deaths per year in the world ${ }^{1}$. Recent studies have evaluated key molecular pathways of $\mathrm{BCa}$, such as RAS-mitogenactivated protein kinase (MAPK) pathway, Wnt/ $\beta$-catenin signaling pathway, and epithelial-mesenchymal transition (EMT) ${ }^{2}$. Coldinducible RNA binding protein (CIRBP) was originally identified in the testis as a mammalian cold shock protein $^{3}$, and is induced by cellular stresses such as UV

Correspondence: Xinghuan Wang (wangxinghuan@whu.edu.cn) 'Department of Urology, Zhongnan Hospital of Wuhan University, Wuhan, China

${ }^{2}$ Department of Biological Repositories, Zhongnan Hospital of Wuhan University, Wuhan, China

Full list of author information is available at the end of the article. These authors contributed equally: Mengxin Lu, Qiangqiang Ge

Edited by I. Amelio radiation, cold and hypoxia ${ }^{3-6}$. CIRBP has been reported to be overexpressed in several human tumors, such as prostate, colon, breast and skin carcinomas ${ }^{7-9}$. Recent mechanistic studies show that CIRBP displayed the ability to bypass replicative senescence through activation of the ERK1/2 signaling pathway in primary mouse embryonic fibroblasts ${ }^{7}$, further evidence for CIRBP's oncogenic function is that CIRBP promotes the development of liver cancer by increasing ROS accumulation and CD133 expression ${ }^{10}$. However, the involvement of CIRBP in BCa has not yet been investigated.

Recent studies describe that the stabilization of mRNAs by specific RNA binding proteins (RBPs) can affect the rate of translation in response to stress ${ }^{4,11}$ or extracellular signals $^{12}$. Recently, several important studies ${ }^{13,14}$ have reported that Y-box binding protein 1, an RNA-binding protein, enhances HIF-1 $\alpha$ protein expression by directly

\section{(c) The Author(s) 2018}

(c) (i) Open Access This article is licensed under a Creative Commons Attribution 4.0 International License, which permits use, sharing, adaptation, distribution and reproduction c. in any medium or format, as long as you give appropriate credit to the original author(s) and the source, provide a link to the Creative Commons license, and indicate if changes were made. The images or other third party material in this article are included in the article's Creative Commons license, unless indicated otherwise in a credit line to the material. If material is not included in the article's Creative Commons license and your intended use is not permitted by statutory regulation or exceeds the permitted use, you will need to obtain permission directly from the copyright holder. To view a copy of this license, visit http://creativecommons.org/licenses/by/4.0/. 
binding to and activating translation of HIF-1A messages. CIRBP is an RNA-binding protein and binds specifically to the $3^{\prime}$-untranslated regions ( $3^{\prime}$-UTR) of many target mRNAs ${ }^{4,15,16}$, and affects their post-transcription expression. It was described that CIRBP can increase in response to hypoxia by a HIF- $1 \alpha$-independent mechanism $^{6}$, moreover, our results demonstrate that CIRBP can increase HIF- $1 \alpha$ expression in BCa. In addition, Yang et al. reported that HIF-1A is one of CIRBP-targeted transcripts in the UniGene $3^{\prime}$-UTR data base ${ }^{15}$. These results indicate that CIRBP may increase mRNA stability and protein translation of HIF- $1 \alpha$ by binding to the $3^{\prime}-$ UTR of its mRNA transcripts.

In human cancers, overexpression of HIF-1A in tumor tissue compared with normal tissue has been revealed in many human cancers ${ }^{17-20}$. Besides, Theodoropoulos et al. reported that HIF-1A was overexpressed in $\mathrm{BCa}$, and its overexpression was related to poor prognosis ${ }^{21}$. Moreover, our qRT-PCR results using 20 pairs of $\mathrm{BCa}$ tissues also showed that HIF-1A is upregulated in BCa tissues. As a key regulator, HIF- $1 \alpha$ target genes play major roles in critical aspects of tumor biology. As a well-known HIF-1 $\alpha$ target gene, VEGF expression can be induced by HIF-1 $\alpha$ in many cancers, which can enhance vascular permeability, stimulate angiogenesis, and increase local tissue oxygenation $^{22-24}$. Furthermore, HIF-1 $\alpha$ target genes can promote EMT, which include snail, transcription factor 3, and zinc finger E-box-binding homeobox 1 and 2 (ZFHX1A, and ZFHX1B) $)^{25,26}$. Gene-expression array demonstrate that more than 1,000 genes are transactivated by HIFs in response to hypoxia ${ }^{27}$, Manalo et al. reported that the PTGIS is an important target gene of HIF- $1 \alpha$ and may be directly regulated by HIF- $1 \alpha^{28}$.

PTGIS encodes prostaglandin synthase (prostaglandin I2 synthase), catalyzes the conversion of prostglandin $\mathrm{H} 2$ to prostacyclin (prostaglandin I2, PGI2) ${ }^{29}$. In cancers, many studies displayed that PGI2 can suppress proliferation and metastasis by activating peroxisomeproliferator-activated receptors (PPARs) ${ }^{30-32}$. Our microarray analysis (GEO accession No. GSE76211) using $\mathrm{BCa}$ tissues and normal bladder epithelium indicated that PTGIS is significantly downregulated in BCa tissues, and further studies shows that overexpression of HIF-1A in $\mathrm{BCa}$ cell line can decrease the expression of PTGIS by binding the hypoxia response element (HRE) of its promoter. Increasing evidences show that the methylation of CpG sites in promoter regions affects the regulation of gene expression by HIF- $1 \alpha$. It has been reported that methylation free HIF-1 $\alpha$ Binding Site is required in several genes expression regulation by HIF- $1 \alpha$, such as MUC17, S100A4 and Erythropoietin ${ }^{33-35}$. Recent researches reported that PTGIS promoter is hypermethylated in colorectal cancer ${ }^{36,37}$, MethHc database (http://methhc. mbc.nctu.edu.tw/php/index.php) shows that PTGIS has a high DNA methylation levels in BCa. Therefore, we speculate that DNA methylation could be the reason that PTGIS expression is downregulated by HIF- $1 \alpha$ but not upregulated by it in $\mathrm{BCa}$ cell line.

\section{Materials and methods \\ Human bladder tissue samples}

Bladder cancer and paracancerous tissue samples $(n=$ 20) were obtained from patients after surgery at Zhongnan Hospital of Wuhan University, informed consent was collected from all subjects, and the histology diagnosis was confirmed pathologically by two pathologists independently. All the tissues were immediately stored in liquid nitrogen or fixed in 4\% PFA after collection from the operation room. The informed consent was signed by all subjects. All specimens collection and treatment were carried out in accordance with the approved guidelines according to the Ethics Committee at Zhongnan Hospital of Wuhan University (approval number: 2015029).

\section{Plasmid construction}

CIRBP cDNA (518bp) and PTGIS cDNA (1502 bp) were polymerase chain reaction amplified from human $\mathrm{BCa}$ cell lines cDNA library and both cloned into 2xFlagpcDNA3 empty vector, using CIRBP forward primer sense EcoR1 5'-CGGAATTCATGGCATCAGATGAAGGC-3'; CIRBP reverse primer sense Xho1 5'-AACTCGAGTT ACTCGTTGTGTGTAGCGT-3'; PTGIS forward primer sense BamH1 5'-TAGGATCCATGGCTTGGGCCGCGC T-3'; PTGIS reverse primer sense EcoR5 5'-TCGATATCTCATGGGCGGATGCGGTAGCG-3'; The DNA sequence was verified by sequencing. p3xFlag-CMV-14HIF-1A plasmid was a gift from Dr. Zhongqiang Guo (Department of Urology, Zhongnan Hospital of Wuhan University). $p$ GL4.10-PTGIS promoter plasmid and $p$ GL4.10-PTGIS promoter mut plasmid were purchased from Obio Technology Corp. Ltd. (Shanghai, China). Biotin-label mRNA transcripts were purchased from GenePharma biotech company (Shanghai, China).

\section{Cell culture, transfections, and stable cell lines selection}

Human BCa cell lines 5637, UM-UC-3, T24 and human epithelial SV40 immortalized uroepithelium cell line SVHUC-1 were obtained from the Stem Cell Bank, Chinese Academy of Sciences in Shanghai, China. Human BCa cell lines BIU-87 were purchased from the Procell Co., Ltd. in Wuhan, China. The $\mathrm{BCa}$ cell lines were identified by the China Centre for Type Culture Collection in Wuhan, China. 5637, T24, SV-HUC-1, BIU-87 Cells were cultured in RPMI-1640 medium (Gibco, China) and UM-UC-3 cells was cultured in DMEM high glucose medium (Gibco, Australia), with 10\% fetal bovine serum (FBS) (Gibco, Australia) in $5 \% \mathrm{CO}_{2}$ at $37^{\circ} \mathrm{C}$. 
For transfection, cells were transfected with plasmids or siRNA oligonucleotides using Lipofectamine 2000 transfection reagent according to the manufacture's protocol. The sense sequence of CIRBP-siRNA (siCIRBP) / CIRBPshRNA (shCIRBP) was 5'-CCAGAGAUCUCGGGGAU UUTT-3', the sense sequence of control-siRNA (NC) / control-shRNA (NC) was 5'-UUCUCCGAACGUGUCACGUTT- ${ }^{\prime}$, and the sense sequence of HIF-1A-siRNA (si HIF-1A) was 5'-GCCGCUCAAUUUAUGAAUATT3', the sense sequence of PTGIS-siRNA (siPTGIS) was 5'GGCUGAAGAAUUACAACAUTT-3'.

For stable cell line selection, UM-UC-3 cells were infected with lentiviral-CIRBP-shRNA and lentiviralcontrol-shRNA $(L V-N C)$ for $24 \mathrm{~h}$, and treated by $5 \mu \mathrm{g} / \mathrm{ml}$ puromycin (Sigma, USA) for 7 days for further selection.

Cell proliferation, clonogenic survival assay, and migration

Cell proliferation was performed by MTT assay. After transfection for $48 \mathrm{~h}, \mathrm{BCa}$ cells were plated into 96-well plates (3000 cells per well) in $200 \mu$ l suspension medium (RPMI-1640 medium or DMEM high glucose medium) to grow for another 5 days. After indicated days, $20 \mu \mathrm{l} \mathrm{MTT}$ solution $(5 \mathrm{mg} / \mathrm{ml})$ was added into each well and incubated for $4 \mathrm{~h}$ at $37^{\circ} \mathrm{C}$. Absorbance at $490 \mathrm{~m}$ was measured by Microplate reader (Cat. \#SpectraMax M2, Molecular Devices, USA).

For clonogenic survival assay, $48 \mathrm{~h}$ after transfection, distinct BCa cells were seeded in 6-well plates (1000 cells per well) and grew into colonies for approximately 14 days. The cells were washed gently twice with PBS and fixed in PBS with $4 \%$ freshly made paraformaldehyde for $30 \mathrm{~min}$ and then stained by crystal violet. The number of colonies was counted and statistically analyzed.

The transwell migration assay was conducted using 24well plates transwell chamber system (Corning, USA) with $8.0 \mu \mathrm{m}$ pore size. Briefly, $48 \mathrm{~h}$ after transfection, BCa cells were seeded $\left(3 \times 10^{\wedge} 4\right.$ cells per chamber $)$ in $200 \mu \mathrm{l}$ serumfree medium in the upper transwell chamber (Corning, USA), while the lower chamber was filled with $600 \mu \mathrm{l}$ medium containing 10\% FBS. After incubation for $24 \mathrm{~h}$ at $37^{\circ} \mathrm{C}$, the cells on the upper chamber of filter were removed, and the cells on the lower chamber were fixed with $4 \%$ PFA and stained by $0.1 \%$ crystal violet. Then the chambers were placed under an inverted phase contrast microscope, the number of cells was counted and photographed in three random fields.

\section{RNA isolation and quantitative real-time PCR}

Total RNA was isolated by HiPure Total RNA Mini Kit (Magen, China) from BCa cells and bladder tissues, quantity of isolated RNA was measured by NanoDrop. The reverse transcription reaction was performed with ReverTra Ace qPCR RT Kit (Toyobo, China). $1 \mu$ of the resulting cDNAs were used as templetes for each reaction of the RT-PCR with iQTM SYBR ${ }^{\circledast}$ Green Supermix (BioRad, USA) in a final volume of $20 \mu$ l. Primer sequences are listed in Supplementary Table S1. Fold enrichment was calculated with the $-\Delta \Delta \mathrm{Ct}$ method relative to GAPDH.

\section{Total protein isolation, Western blot, and immunofluorescence staining}

$\mathrm{BCa}$ cells were washed with PBS for three times and lysed by RIPA buffer containing protease inhibitor and phosphatase inhibitor (Sigma-Aldrich, USA) for $30 \mathrm{~min}$ on ice. The cell lysates were then centrifuged at $13,000 \times g$ for $15 \mathrm{~min}$. The supernatants were collected and protein concentration was determined by Bradford protein assay (Bio-Rad, Germany). The isolated total protein samples $(25-30 \mu \mathrm{g})$ were separated by $6-15 \%$ SDSPAGE and subjected to Western blot analysis. The immunoreactive bands were visualized using an enhanced chemiluminescence kit (Bio-rad, USA) and detected by Molecular Imager Chemi Doc XRS ${ }^{+}$Imaging system (Bio-rad, USA). The primary antibodies and secondary antibodies were listed in Supplementary Table S2. Immunofluorescence staining for $\mathrm{BCa}$ cells seeded on $12 \mathrm{~mm}$ coverslips and fixed tissue samples were accomplished by Biofavor Biotech company (Wuhan, China), immunofluorescence staining was analysed by using a Confocal microscope system (Nikon $\mathrm{C}^{+}$Confocal Microscope, Japan).

\section{mRNA stability assay, cycloheximide (CHX) assay, RNA- binding protein immunoprecipitation assay, and biotin pull-down assay}

In order to examine the effects of CIRBP on the stability of the mRNAs of HIF-1A, 24h after transfection, Cells were pretreated with $\mathrm{CoCl}_{2}(100 \mu \mathrm{M}$, Sigma c8661) for 4 $\mathrm{h}$, then Actinomycin D (15 $\mu \mathrm{g} / \mathrm{ml}$, Abcam ab141058) was added to inhibit transcription. Total RNA was collected at specific time points of $0,2,4,6,8 \mathrm{~h}$, and RT-PCR detected mRNA levels of HIF-1A.

In order to examine the effects of CIRPR on HIF- $1 \alpha$ protein stability, $24 \mathrm{~h}$ post-transfection, Cells were pretreated with $\mathrm{CoCl}_{2}(100 \mu \mathrm{M})$ for $4 \mathrm{~h}$, then cycloheximide $(200 \mu \mathrm{g} / \mathrm{ml}$, MCE, HY-12320) was added inhibit protein synthesis. Total protein samples were collected at specific time points of $0,2,4 \mathrm{~h}$, and Western blot detected protein levels of HIF- $1 \alpha$.

For RNA-Binding Protein Immunoprecipitation (RIP) assay, 24 h after transfection with CIRBP overexpression plasmid, the cells on the plates were washed twice with $5 \mathrm{ml}$ of ice-cold PBS, then lysed with $1 \mathrm{ml}$ lysis buffer (150 mM NaCl, 1\% IGEPAL CA-603, 0.5\% DOC, 0.1\% SDS, $50 \mathrm{mM}$ Tris $100 \mathrm{U} / \mathrm{ml}$ Rnasin, $1 \mathrm{~mm}$ PMSF) for 10 $\mathrm{min}$, the cell lysates were then centrifuged at $16,000 \times g$ for $10 \mathrm{~min}$ and the supernatants were collected. $500 \mu \mathrm{l}$ supernatants were incubated with $100 \mu$ l Protein G Sepharose beads (GE Healthcare Life Sciences, USA) with 
rotating for overnight which had been pre-coated with $40 \mu \mathrm{g}$ of either anti-CIRBP or Normal rabbit anti-IgG1 for $6 \mathrm{~h}$. The beads were washed five times $(0.5 \mathrm{ml}$ wash, $5 \mathrm{~min}$ each) with Wash buffer (50 mM Tris- $\mathrm{HCl}, 150 \mathrm{mM} \mathrm{NaCl}$, $1 \mathrm{mM} \mathrm{MgCl} 2,0.05 \% \mathrm{NP}-40$ and $100 \mathrm{U} / \mathrm{ml}$ Rnasin), bound proteins were then digested by proteinase $\mathrm{K}$ Buffer (adding $0.1 \%$ SDS and $0.5 \mathrm{mg} / \mathrm{ml}$ proteinase $\mathrm{K}$ ) at $55^{\circ} \mathrm{C}$ for $30 \mathrm{~min}$. Total RNA was isolated by chloroform extraction and resuspended in DEPC-treated water. RTPCR analyzed the mRNA levels of HIF-1A, then the IP efficiency was calculated by percent of input (Percent Input $=2 \% \times 2$ (CT $2 \%$ Input Sample-CT IP Sample) .

Biotin pull-down assays were performed by incubating $40 \mu \mathrm{g}$ of cytoplasmic fractions with $50 \mathrm{pmol}$ of biotin-label mRNA transcripts for $1 \mathrm{~h}$ at room temperature, and the ribonucleoprotein complexes were then isolated with $50 \mu \mathrm{l}$ of streptavidin-conjugated Dynabeads (Invitrogen, \# 11205D). The presence of CIRBP in the pull-down pellets was verified using Western blot analysis.

\section{Chromatin immunoprecipitation (ChIP) assay and luciferase reporter assay}

The ChIP assay was performed with Simple CHIP ${ }^{\circledR}$ Plus sonication Chromation IP Kit (\#56383, Cell Signaling Technology, USA) and carried out according to the manufacturer's protocol. Briefly, $24 \mathrm{~h}$ after transfection with p3xFlag-CMV-14-HIF-1A plasmid or empty vector, cells were Cross-linked in $37 \%$ formaldehyde, and scraped into cold buffer, then continued with nuclei preparation and chromatin digestion. DNA fragment size was determined by electrophoresis on a $1 \%$ agarose gel (approximately 100-1000 bp). Then cross-linked chromatin was incubated with $2 \mu \mathrm{g}$ anti-FLAG or normal rabbit IgG for overnight at $4{ }^{\circ} \mathrm{C}$ with rotation. After washing the protein $G$ magnetic beads with low salt washing buffer and high salt washing buffer, Chromatin was eluted from Antibody/Protein G Magnetic Beads, detected by quantitative realtime PCR and calculate the IP efficiency (Percent Input $=2 \% \times 2^{(\mathrm{CT}} 2 \%$ Input Sample-CT IP Sample) $)$, Primer sequences for PTGIS promoter are listed in Supplementary Table $\mathrm{S} 3$.

For the luciferase reporter assay, cells were seeded onto 24-well plates and cotransfected with $500 \mathrm{ng}$ of luciferase reporter plasmids (pGL4.10-PTGIS promoter vector, pGL4.10-PTGIS promoter mut vector or pGL4.10 base vector), and $500 \mathrm{ng}$ of HIF-1A expression vector or empty vector. After $24 \mathrm{~h}$, luciferase activity was measured with Luc-Pair ${ }^{\mathrm{TM}}$ Duo-Luciferase Assay Kit 2.0 (GeneCopoeial lnc, USA) according to its protocol.

\section{Xenograft model and pulmonary metastasis model}

Male BALB/c-nu mice (4-weeks old) were purchased from Beijing Vital River Laboratory Animal Technology Co., Ltd. (Beijing, China), and maintained in laboratory animal facility of Zhongnan Hospital of Wuhan University. After adaptive feeding for a week, xenograft models were established by subcutaneously inoculating $1 \times 10^{6}$ UM-UC-3 $L V$-NC cells or $L V$-CIRBP sh cells diluted in $100 \mu \mathrm{PBS}(\mathrm{n}=4)$, while pulmonary metastasis models were established by tail intravenous injecting $1 \times$ $10^{6}$ cells diluted in $100 \mu \mathrm{l}$ PBS $(n=3)$. 6 weeks post injection, the mice were sacrificed and the tumors were taken out and weighed, meanwhile the tumor volume was measured every 6 days (tumor volume $=$ length $\times$ width $\times$ $0.5 \mathrm{~mm}^{3}$ ). The fluorescence of pulmonary metastasis tumor was measured by FUSION FX7 Spectra Imaging system (Vilber, France).

\section{Statistical analyses}

Data were expressed as mean \pm SD from three individual experiments, statistical analyses were performed with SPSS Statistics 22.0. Unpaired 2-tailed $T$-test and one-way analysis of variance were used to evaluate statistical significance of differences of data, $p<0.05$ were considered significant.

\section{Results \\ CIRBP acts as an oncogene during $\mathrm{BCa}$ progression}

To investigate the CIRBP expression level in $\mathrm{BCa}$, CIRBP mRNA was analyzed by qRT-PCR analysis, indicating no significant difference in the BCa tissues compared with the paired paracancerous tissues $(n=21$, Supplementary Figure S1A). We next analyzed the association between the CIRBP mRNA levels and the tumor stage of these $\mathrm{BCa}$ tissues, indicating that CIRBP expression levels were positively correlated with the $\mathrm{T}$ stage in BCa (Fig. 1a). Furthermore, Western blot analyzed the protein level of CIRBP in 14 pairs of $\mathrm{BCa}$ tissues and paracancerous tissues, CIRBP protein upregulation was observed in 8 of 14 (57\%) BCa tissues (Fig. 1b). To further confirm CIRBP protein upregulation, immunofluorescence staining was performed, CIRBP was observed in the nucleus, and it revealed strong increase of CIRBP protein in the $\mathrm{BCa}$ tissues (Fig. 1c). In addition, we detected the CIRBP protein levels in four BCa cell lines (5637, UM-UC-3, T24 and BIU-87) and immortalized normal uroepithelial cell line (SV-HUC-1), exhibiting an upregulation tendency of CIRBP in BCa cell lines (Fig. 1d). To obtain further insight into the function of CIRBP, we searched the gene function enrichment by using miRNA Cancer MAP database (http://cis.hku.hk/ miRNACancerMAP/index.php). As shown in Fig. 1e, f, CIRBP was significantly enriched in several pathways, such as RNA degradation, cell cycle, adherens junction.

To study the effects of CIRBP on the biological behaviors of $\mathrm{BCa}$ cells, an siRNA specifically targeting CIRBP was used to knock down CIRBP expression, while an CIRBP gene overexpression plasmid was used to 


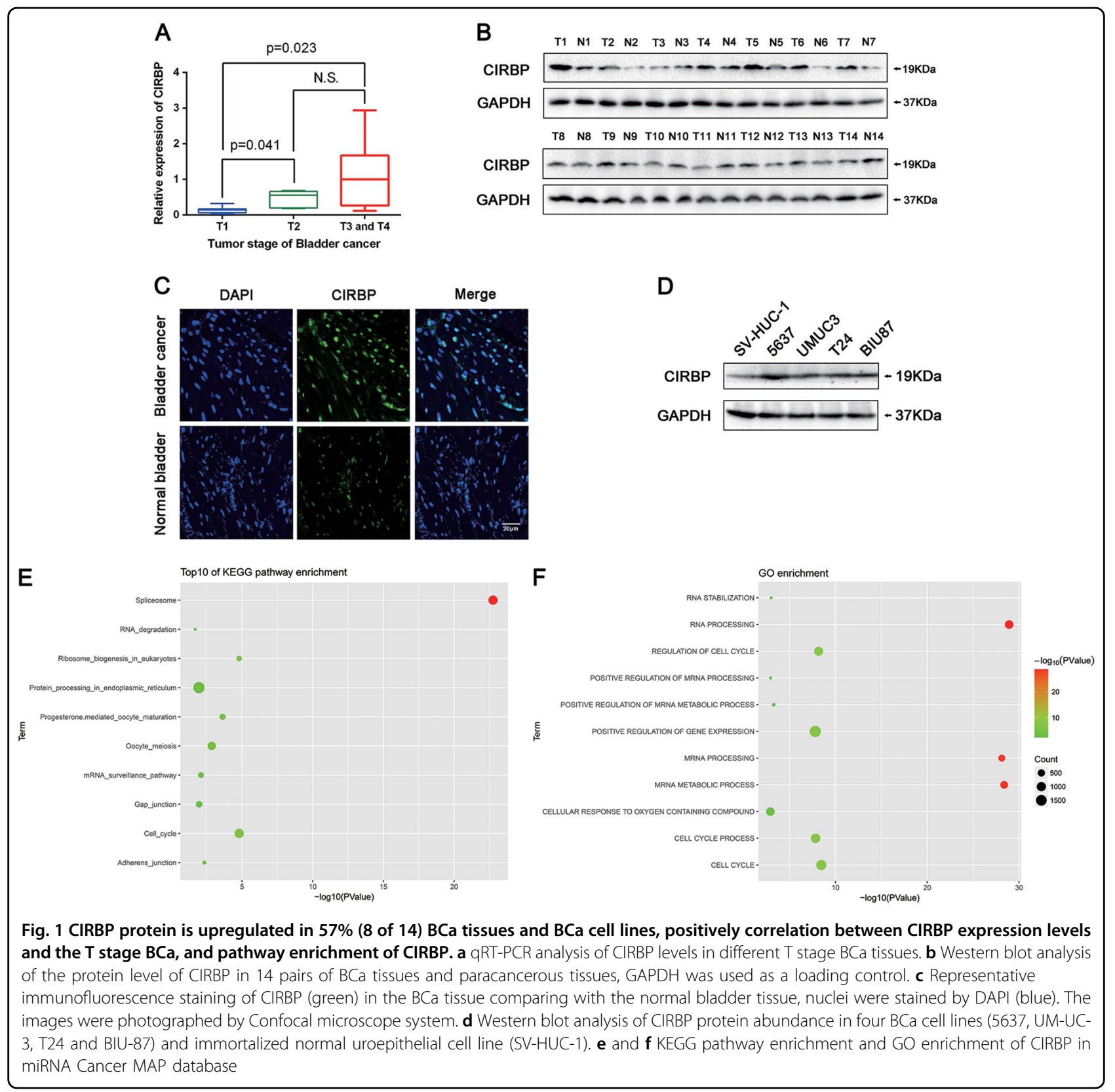

upregulate CIRBP expression in two BCa cell lines (UM$\mathrm{UC}-3$ and 5637). Western blot analysis verified the overexpression and knockdown efficiencies, and qRT-PCR analysis demonstrated the same results in mRNA levels (Supplementary Figure S1B). Next, we evaluated the cell proliferation and migration abilities. MTT assay showed that CIRBP-siRNA-treated $\mathrm{BCa}$ cells grew significantly slower than the control $\mathrm{BCa}$ cells, conversely, overexpression of CIRBP dramatically promoted cell growth (Fig. 2a). In agreement with its facilitated proliferation function, clonogenic survival assay revealed that the ability of colony formation was significantly decreased in the CIRBP knockdown cells, while increased in CIRBP overexpression cells (Fig. 2b). What's more, transwell migration assay suggested that knockdown of CIRBP in $\mathrm{BCa}$ cells could reduce cell migration and overexpression of CIRBP could induce cell migration (Fig. 2c).

\section{Effects of CIRBP on MAPK signaling pathway and EMT markers}

MAPK pathway plays an important role in various tumor physiological processes, including cell proliferation, cell differentiation and cell survival ${ }^{38,39}$, our transcriptome data also indicated that MAPK signaling pathways play a key role in $\mathrm{BCa}^{40,41}$. CIRBP has been reported to be involved in multiple tumor progression via 


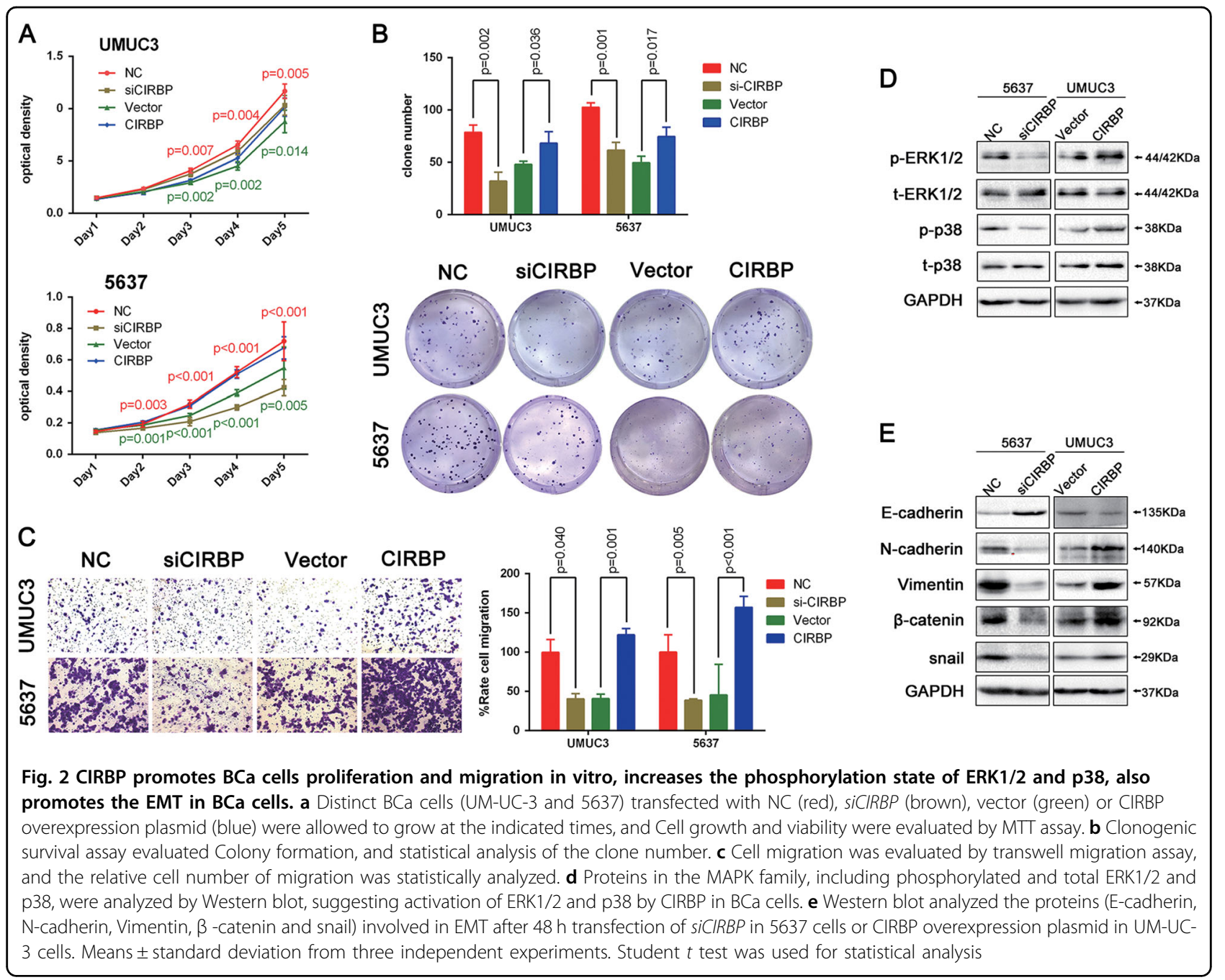

the activation of ERK and p38 MAPK $^{7,42,43}$. Our Western blot analysis showed phosphorylated ERK1/2 (p-ERK1/2) and phosphorylated p38 (p-p38) protein levels were obviously decreased in the si-CIRBP-treated 5637 cells, whereas increased in the CIRBP overexpression UM-UC3 cells (Fig. 2d).

Moreover, because CIRBP could promote cell migration, and pathway enrichment indicated that CIRBP is related with adherens junction, proteins involved in the EMT process were analyzed by Western blot analysis. CIRBP knockdown could suppress the expression of $\mathrm{N}$ cadherin, vimentin, $\beta$-catenin and snail in 5637 cells, reversely, CIRBP overexpression could increase their expression level in UM-UC-3 cells. In addition, knockdown of CIRBP could upregulate the epithelial marker Ecadherin in 5637 cells, and overexpression of CIRBP could reduce E-cadherin protein expression in UM-UC-3 cells (Fig. 2e).

\section{Reduction of CIRBP inhibits BCa growth and pulmonary} metastasis in vivo

To verify the function of CIRBP in vivo, lentiviralCIRBP-shRNA and lentiviral-control-shRNA (LV-NC) stable cell line were established, after selection with puromycin for 7 days, the green fluorescence of stable cell lines was showed in Fig. 3a, qRT-PCR analysis validated the Efficiency of CIRBP knockdown by lentiviral-CIRBPshRNA (Fig. 3a). Next, as shown in Fig. 3b, c, xenograft models were construct, the $L V$-shCIRBP group grew significantly slower than the control group in vivo, and the mean tumor weight was significantly lower in the $L V$ shCIRBP group than in the $L V-N C$ group (Fig. 3c). The dissected neoplasms tissues were embedded into paraffin, the Hematoxylin and Eosin staining (HE staining) results suggested the reduced number of tumor cells in the $L V$ shCIRBP group, and the immune-fluorescence staining displayed that CIRBP expression is weaker in the $L V$ - 


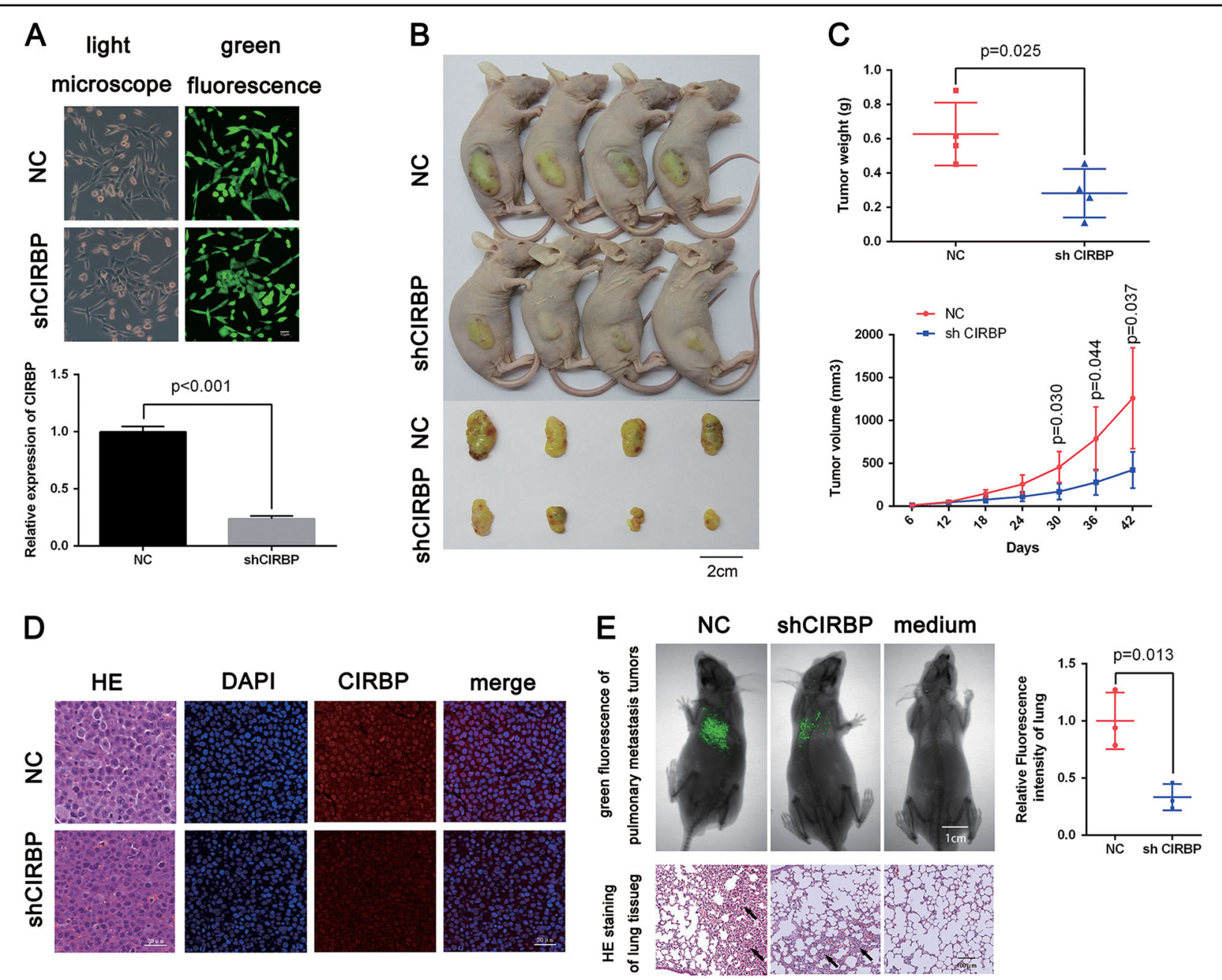

Fig. 3 Knockdown of CIRBP inhibits BCa growth and migration in vivo. a The green fluorescence of stable cell lines, and qRT-PCR analysis verification of CIRBP knockdown efficiency by lentiviral-CIRBP-shRNA. b Xenograft models $(n=4)$ were established by subcutaneously inoculating $L V$ NC cells or LV-shCIRBP cells and allowed to grow for 6 weeks, then the mice were sacrificed and the tumors were taken out and weighed. $\mathbf{c}$ tumor volume measurement and tumor weight. $\mathbf{d}$ Representative H\&E staining and immunefluorescence staining of xenograft tumors from the tumorbearing mice of the LV-NC group and LV-shCIRBP group, indicating the downregulation of CIRBP (red) in LV-shCIRBP group. Nuclei were stained by DAPI (blue). e Pulmonary metastasis models $(n=3)$ were established by tail intravenous injecting LV-shCIRBP UM-UC-3 cells or LV-NC UM-UC-3 cells, the fluorescence intensity of pulmonary metastasis tumor was measured to evaluate the migration capacity. Representative H\&E staining of lung tissues indicating the pulmonary metastasis tumors (pointed by the arrows). Statistical analysis of the fluorescence intensity was calculated using $T$ test. Means \pm standard deviation from three independent experiments

shCIRBP group compared with the $L V-N C$ group (Fig. 3d).

In the same way, for the purpose of investigating the effect of CIRBP knockdown on BCa metastasis in vivo, pulmonary metastasis models were established by tail intravenous injecting $L V$-shCIRBP UM-UC-3 cells or $L V$ $N C$ UM-UC-3 cells, after 4 weeks of injection, the fluorescence of pulmonary metastasis tumor was measured to evaluate the migration capacity. As exhibited in the Fig. 3e, CIRBP knockdown could significantly suppress migration in vivo in contrast with the $L V-N C$ group, the HE staining displayed the pulmonary metastasis tumors (pointed by the arrows).
CIRBP increases mRNA stability and protein translation of HIF-1a in BCa cell line

To investigate the relationship between CIRBP and HIF- $1 \alpha$ in BCa, we first evaluated whether HIF- $1 \alpha$ could modulate the expression of CIRBP, qRT-PCR analysis showed that HIF-1A overexpression have no obvious effect on the mRNA level of CIRBP (Supplementary Figure S1C). Next, the study addressed the effects of CIRBP on HIF- $1 \alpha$ expression, as shown in the Fig. 4a, qRT-PCR analysis showed that both CIRBP knockdown and CIRBP overexpression had no significant effects on the mRNA levels of HIF-1A. Moreover, Western blot analysis showed that transfection of $s i$-CIRBP could robustly downregulate 


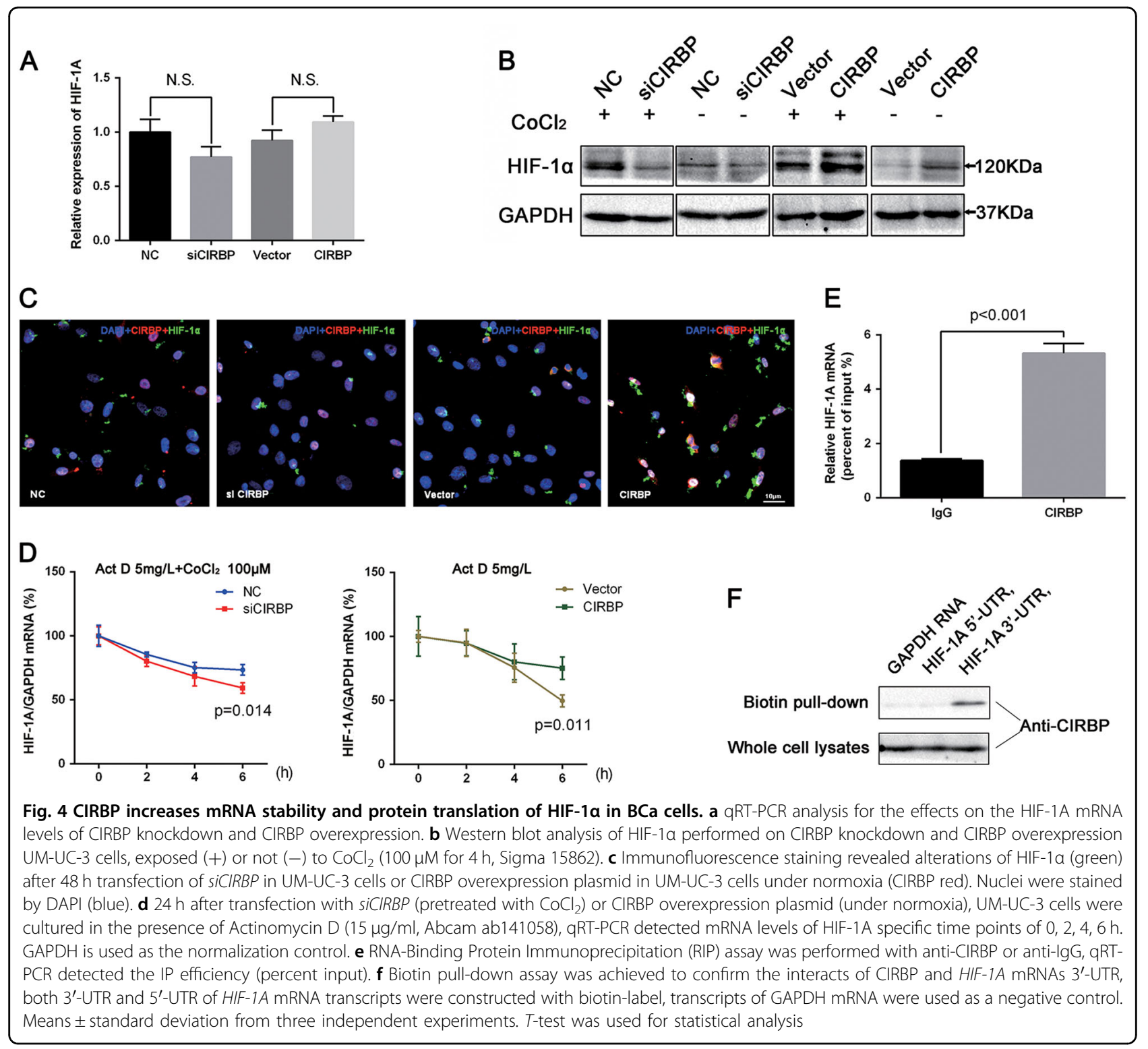

HIF- $1 \alpha$ expression, and overexpression of CIRBP could significantly upregulate HIF- $1 \alpha$ expression, both under normoxia and hypoxia (Fig. 4b), the same results were also revealed by immunofluorescence staining under normoxia (Fig. 4c).

UniGene database indicated that HIF-1A is one of CIRBP-targeted transcripts ${ }^{15}$, and pathway enrichment indicated that CIRBP was significantly enriched in RNA degradation and RNA stabilization. To determine whether CIRBP upregulate HIF- $1 \alpha$ expression through binding to the $3^{\prime}$-UTR and increases its mRNA stability, we first performed mRNA stability experiments in the presence of actinomycin D (a transcriptional inhibitor, $15 \mu \mathrm{g} / \mathrm{ml}$ ), as displayed in Fig. 4d, under the hypoxia conditions (pretreated with $\mathrm{CoCl}_{2} 100 \mu \mathrm{M}$ for $4 \mathrm{~h}$ ), the results demonstrated that transfection with $s i$-CIRBP reduced the stability of HIF-1A mRNA. While in absence of $\mathrm{CoCl}_{2}$, CIRBP overexpression could increase HIF-1A mRNA stability strikingly. Next, RNA-Binding Protein Immunoprecipitation (RIP) assay was used to demonstrate that CIRBP could bind to HIF-1A mRNA (Fig. 4e). Furthermore, biotin pull-down assay was achieved to confirm the interacts of CIRBP and HIF-1A mRNAs $3^{\prime}$-UTR, as shown in Fig. 4f, substantial amounts of CIRBP were associated with the HIF-1A mRNA transcripts 3'-UTR, nevertheless, both the 5'-UTR of HIF-1A mRNA transcripts and the negative control transcripts failed to pull down CIRBP. In addition, in order to investigate the effects of CIRBP on HIF- $1 \alpha$ protein stability, cycloheximide (CHX) assay was performed. As shown in 
Supplementary Figure S1D, CIRBP knockdown had no obvious effects on the HIF- $1 \alpha$ protein degradation.

\section{PTGIS is a HIF-1a target gene in BCa}

As a master regulator in hypoxic cancer progression, HIF- $1 \alpha$ activates transcription of many genes ${ }^{23}$, two publications of microarray hybridization analysis have reported that PTGIS is an important target gene of HIF$1 \alpha^{28,44}$, and our microarray analysis (GEO accession no. GSE76211) indicate that PTGIS is a significantly downregulated gene in $\mathrm{BCa}$ tissues compared with normal bladder tissues. Therefore, our following studies focused on the regulatory effects of HIF- $1 \alpha$ on PTGIS expression in BCa. In the same way, we first detected the mRNA levels of HIF-1A and PTGIS in the BCa tissues $(n=20)$, the results of qRT-PCR analysis displayed that HIF-1A is significantly upregulated, while PTGIS is remarkably downregulated in the $\mathrm{BCa}$ tissues (Fig. 5a). Furthermore, to detect the transcription effects of HIF- $1 \alpha$ on PTGIS expression, a HIF-1A gene overexpression plasmid was used, as shown in Fig. 5b, overexpression of HIF- $1 \alpha$ could suppress the expression of PTGIS both in mRNA level and protein level, immunofluorescence staining also proved the same conclusion. In addition, $H I F-1 A$ knockdown with a siRNA specifically targeting $H I F-1 A$ could increase the PTGIS mRNA level (Supplementary Figure S1E), the overexpression and knockdown efficiencies were validated by qRT-PCR (Supplementary Figure S1E).

As a key transcription factor of the adaptive response to hypoxia, HIF- $1 \alpha$ transcriptional regulates many genes through binding to core DNA sequence 5'-RCGTG-3' within the HRE of target gene promoters ${ }^{45}$. We next predicted an HRE binding site (5'-ccACGTGc-3') of HIF$1 \alpha$ on PTGIS promoter region through using GCBI database (https://www.gcbi.com.cn/gclib/html/index), in addition, as shown in Fig. 5 d, the binding site of HIF- $1 \alpha$ provided by the JASPAR database (http://jaspardev. genereg.net/) supported this conclusion. Furthermore, a ChIP assay was performed to verify this conclusion, after transfection for $24 \mathrm{~h}$, DNA was digested with ultraphonic to a length of approximately 100-1000 bp (Supplementary Figure S1F), and the anti-FLAG was employed to pull down HIF- $1 \alpha$ and its associated DNA fragments. As shown in Fig. 5c, seven pairs of primers were designed to cover the region of the PTGIS promoter, qRT-PCR detected the enrichment results. And the highest enrichment of FLAG-HIF-1A was identified at the P3 region of the PTGIS promoter, followed by the P2 and P4 region, while no significant enrichment was found in other regions (Fig. 5e). Subsequently, luciferase reporter assay was used to evaluate the effects of HIF- $1 \alpha$ on PTGIS promoter activity, as shown in Fig. 5f, overexpression of HIF-1A could obviously enhance the luciferase activity of PTGIS luc compared with empty vector, while mutant of
HRE (5'-ccTGCACc-3') sequence could significantly reduce this induction.

\section{CpG Methylation of PTGIS promoter region plays an important role in transcriptional regulation of HIF-1a}

CpG methylation plays an important regulatory role in the control of gene expression ${ }^{46}$, several researches had reported that the promoter regions methylation affects the regulation of gene expression by HIF- $1 \alpha^{33,34,47}$. HIF$1 \alpha$ could activate transcription of many target genes, especially many oncogenes such as VEGF, erythropoietin, $\mathrm{C}-\mathrm{MYC}$ and OCT4 $4^{23,45}$. However, our results revealed that overexpression of HIF- $1 \alpha$ can suppress the expression of PTGIS in UM-UC-3 cells, so our following studies focused on the relationship between CpG Methylation of PTGIS promoter region and transcriptional regulation of HIF-1 $\alpha$ on PTGIS. As shown in Fig. 6a, MethHc database showed that PTGIS has a high DNA methylation levels in $\mathrm{BCa}$ compared with normal samples, and its DNA methylation was negatively correlated with PTGIS mRNA expression levels in BCa (Supplementary Figure S1G). In addition, UCSC Genome Browser database (http:// genome.ucsc.edu/) indicated that PTGIS gene promoter region has a CpG island (Position: chr20: 4956765749568296), which was displayed in Fig. 5c.

In order to confirm the methylation of PTGIS gene promoter in $\mathrm{BCa}$ cell lines, Bisulfite sequence analyses were performed in three cell lines (SV-HUC-1, UM-UC-3 and 5637), demonstrating that methylation of all $\mathrm{CpG}$ sites of PTGIS gene promoter is more frequent in BCa cell lines (UM-UC-3 and 5637) rather than immortalized normal uroepithelial cell line (SV-HUC-1, Fig. 6b). Apart from downregulation in the $\mathrm{BCa}$ tissues, our qRT-PCR results indicated that PTGIS expression is strikingly reduced in BCa cell lines compared with SV-HUC-1 (Fig. 6c). Next, to further prove the methylation of PTGIS, UM-UC-3 cells were cultured with the demethylating agent 5-Aza-2'-deoxycytidine, and qRT-PCR detected the restoration of PTGIS expression in BCa cells (Fig. 6d). Then we investigated the effects of HIF-1A overexpression on PTGIS in SV-HUC-1 cells, which have a low PTGIS gene promoter methylation level. As shown in Fig. 6e, in contrast with transcriptional suppression in UM-UC-3 cells, transfection of HIF-1A overexpression plasmids could upregulate the expression level of PTGIS. Besides, when UM-UC-3 cells were pre-treated with $5 \mathrm{Aza}$ $(1 \mu \mathrm{M}$ for $24 \mathrm{~h})$, the previous transcriptional suppression would turn to transcriptional activation (Fig. 6f).

\section{PTGIS acts as a tumor suppressor in $\mathrm{BCa}$}

PTGIS has been reported to be a tumor suppressor in many kinds of cancers ${ }^{30-32}$, our results displayed that PTGIS is downregulated both in BCa tissues (Fig. 5a) and in BCa cell lines (Fig. 6c). Similarly, to explore the tumor 


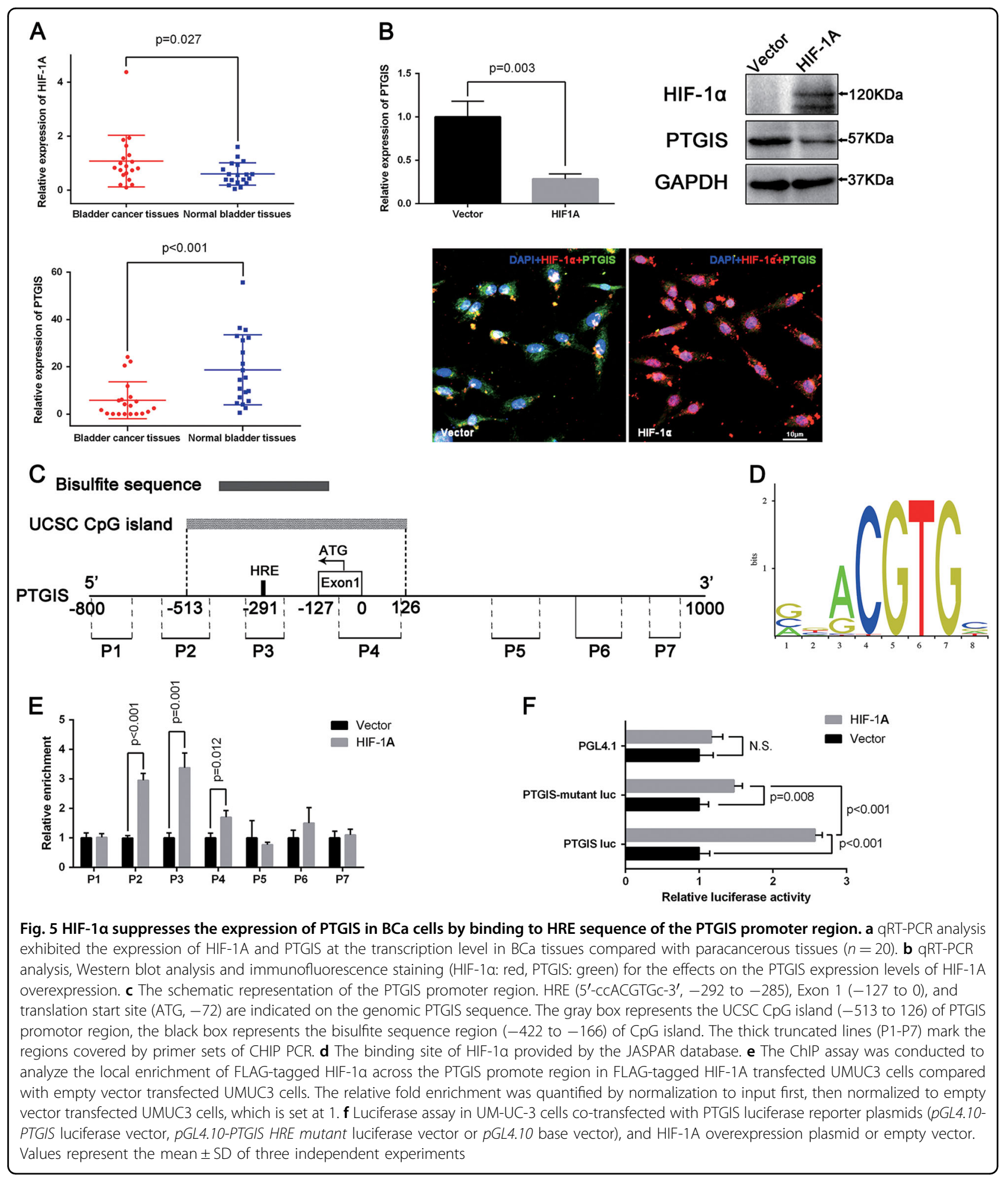

biological functions of PTGIS in BCa, a PTGIS expression plasmid was constructed, and the overexpression efficiency was verified by Western blot (Supplementary Figure $\mathrm{S} 1 \mathrm{H})$. As shown in Fig. 7a, b, MTT assay and clonogenic survival assay demonstrated that overexpression of PTGIS could suppress BCa cells proliferation Moreover, the protein level of PPAR- $\gamma$ was increased by transfection of PTGIS expression plasmids (Fig. 7d). In addition, transwell migration assay showed that PTGIS overexpression could significantly suppress 


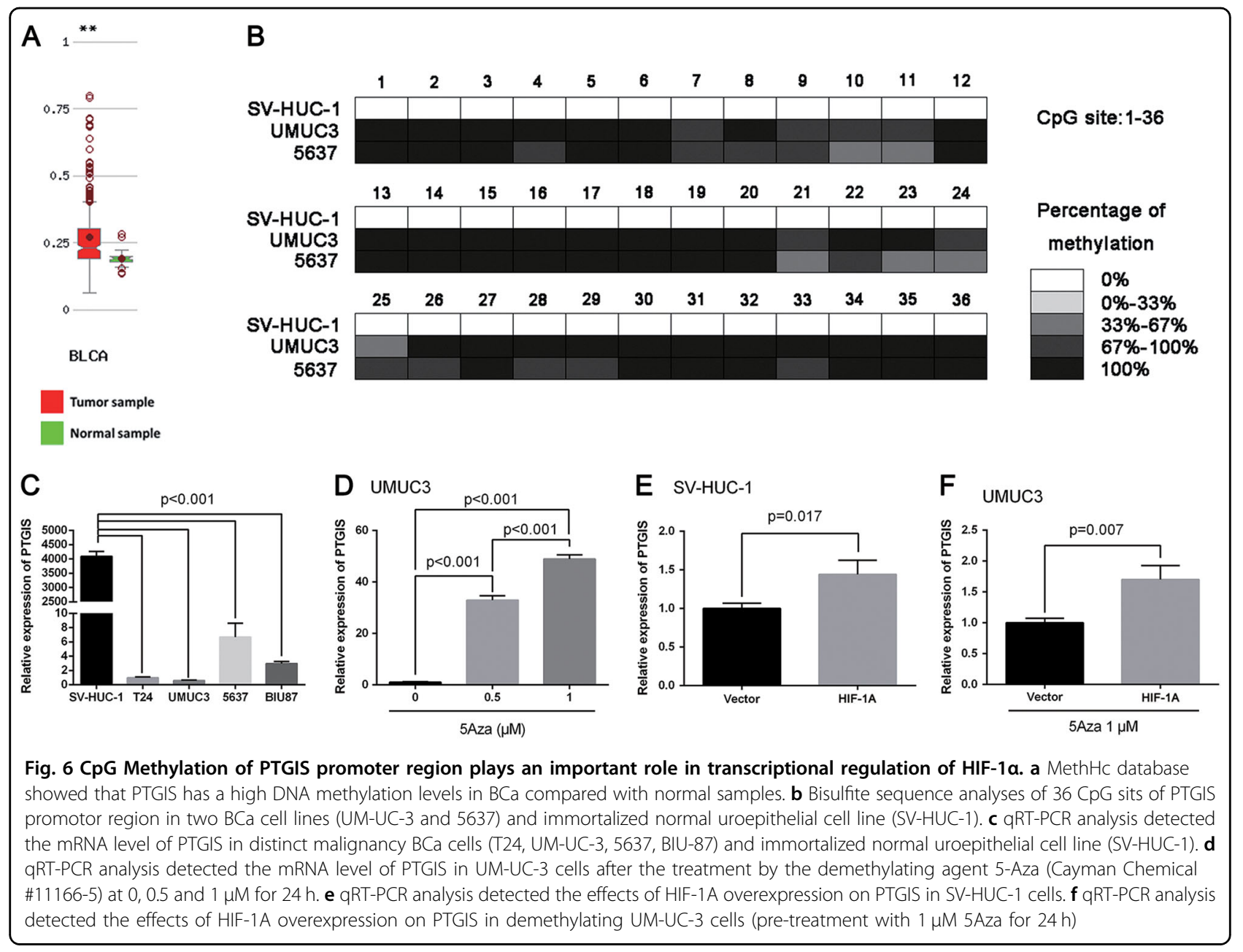

migration of BCa cells (Fig. 7c), while Western blot results further proved that PTGIS overexpression could suppress the EMT in UM-UC-3 and 5637 cell lines (Fig. 7e).

PTGIS knockdown could partially rescue the inhibition of migration and proliferation in siCIRBP treated $\mathrm{BCa}$ cells

Since CIRBP could induce expression of HIF- $1 \alpha$, and HIF- $1 \alpha$ could suppress the expression of PTGIS in BCa cells, we finally explored the regulation relationship of CIRBP on PTGIS expression. As shown in Fig. 7f, knockdown of CIRBP could promote the expression of PTGIS, while overexpression of CIRBP have no obvious suppression effect on PTGIS expression, which may be associated with its low expression in BCa cells, and qRTPCR analysis demonstrated the same results in mRNA levels (Supplementary Figure S1I). In addition, further studies showed that PTGIS knockdown could significantly rescue the inhibition of migration in siCIRBP treated $\mathrm{BCa}$ cells (Fig. $7 \mathrm{~g}$ ), and partial rescue of proliferation inhibition was also observed (Fig. 7h), while PTGIS inhibition have no effect on siCIRBP induced inhibition of clonogenic survival ability in BCa cells (Supplementary Figure S1J). The knockdown efficiency of siPTGIS was validated by Western blot (Supplementary Figure S1K).

\section{Discussion}

Recently, CIRBP has been reported to act as an oncogene in many studies ${ }^{9,10,43,48}$, in this study, we investigated the involvement of CIRBP in $\mathrm{BCa}$. Our results indicated that CIRBP is overexpressed in $57 \%$ BCa tissues and $\mathrm{BCa}$ cell lines, and CIRBP could promote BCa cells proliferation and migration both in vitro and in vivo. Furthermore, we demonstrated that CIRBP expression led to an increase in the phosphorylation state of ERK $1 / 2$ and p38, also promoted the EMT in BCa cells. MAPKs plays a central role in cell proliferation control ${ }^{49}$, our transcriptome data also indicated that MAPK signaling pathways play a key role in $\mathrm{BCa}^{40,41}$, in agreement with our results, evidence of a possible linkage between CIRBP and MAPK signaling pathways comes from two studies, Artero-Castro et al. reported that CIRBP displayed the ability to bypass replicative senescence through activation 


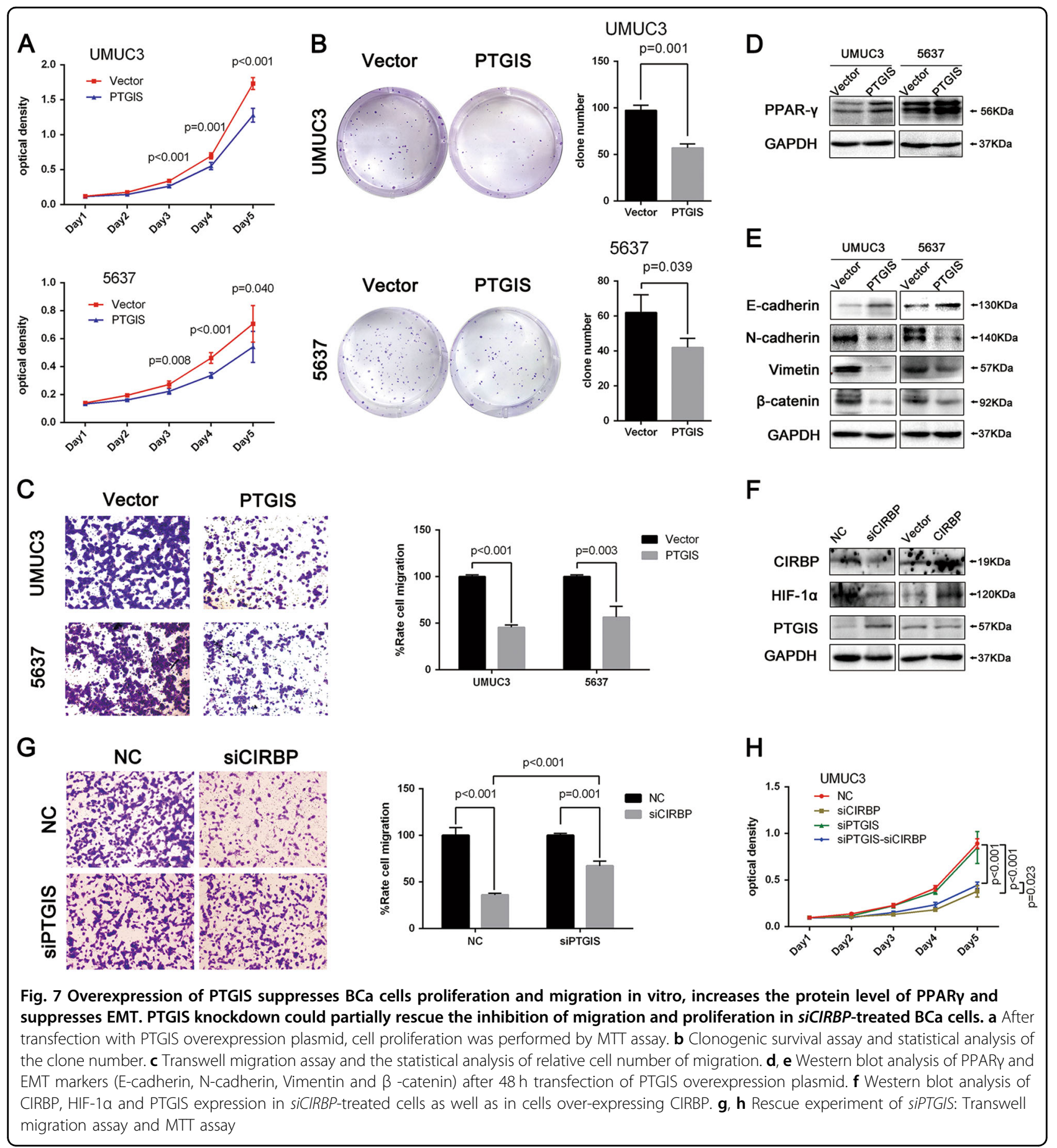

of the ERK1/2 signaling pathway in primary mouse embryonic fibroblasts ${ }^{7}$, the second study by Lee et al. showed that CIRBP could promote epithelial to mesenchymal transition by activating ERK and p38 pathways ${ }^{43}$.

RNA-binding proteins play an essential role in RNA metabolism ${ }^{50}$, CIRBP has been reported to regulate several target genes by specifically binding to the $3^{\prime}$-UTR of its target mRNAs. For instance, CIRBP could bind to the TRX $3^{\prime}$-UTR and stabilize the transcript to increase its translation ${ }^{16}$, another study showed that CIRBP could increase the stability of pro-inflammatory cytokine mRNAs under cold conditions to induce an airway inflammatory response ${ }^{11}$. The results of our study showed that CIRBP could induce HIF- $1 \alpha$ protein production via a CIRBP-mediated increase in mRNA stability in $\mathrm{BCa}$, and 


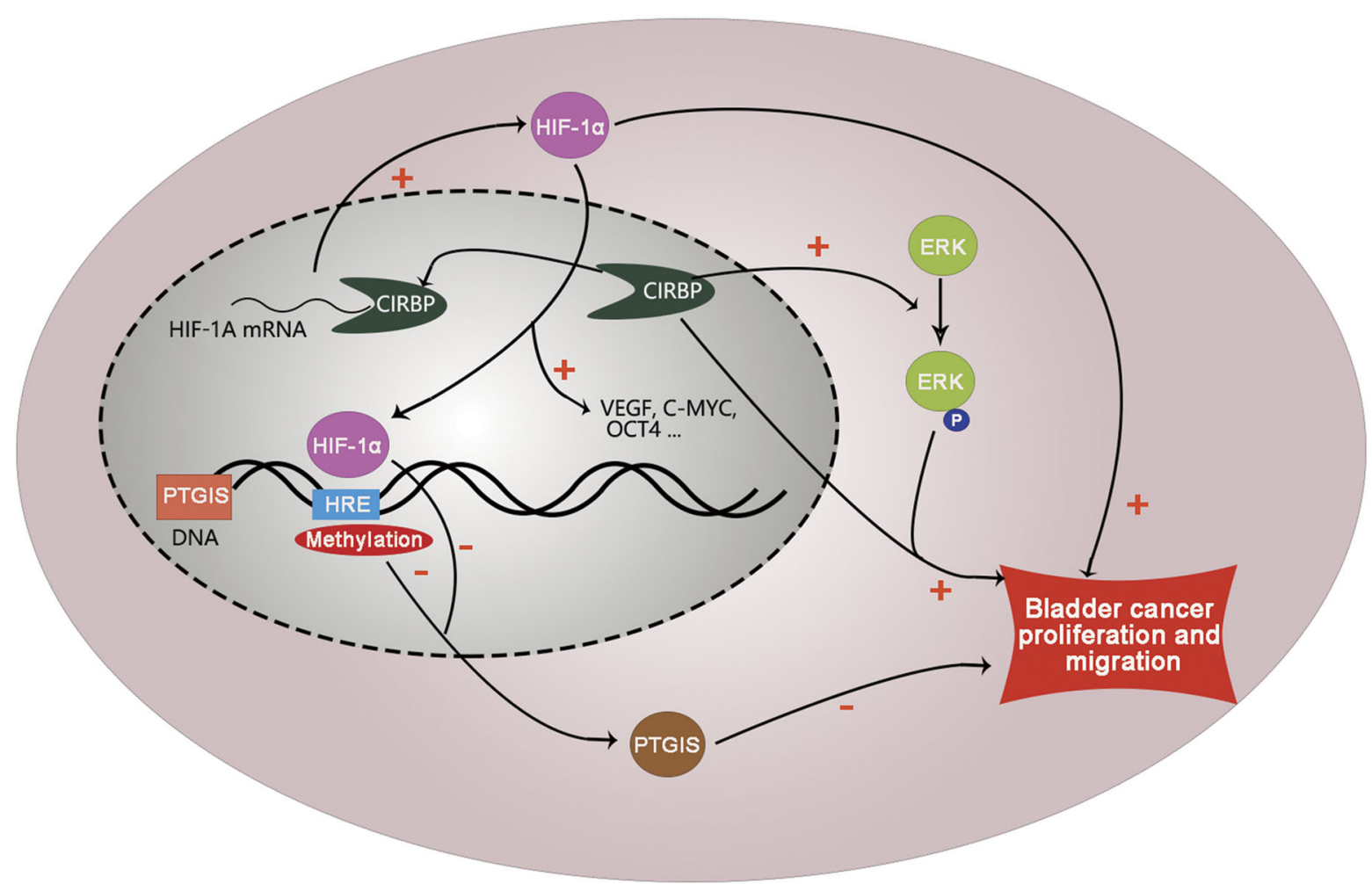

Fig. 8 Mechanism diagram. Our study demonstrated that CIRBP could promote BCa cells proliferation and migration both in vitro and in vivo. Furthermore, CIRBP could increase HIF-1a expression by binding to the $3^{\prime}-U T R$ of HIF-1A mRNA transcripts and increasing its mRNA stability in BCa cell line. HIF-1a could activate transcription of many oncogenes, such as VEGF, C-MYC, and OCT4. Our results showed that PTGIS is a HIF-1a target gene, which could suppress BCa cells proliferation and migration, and overexpression of HIF-1A could suppress the expression of PTGIS in BCa cells

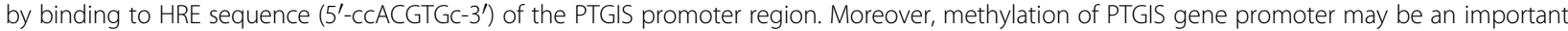
reason why PTGIS expression is downregulated by HIF-1a. And DNA methylation is also an important reason for obvious downregulation of PTGIS in $\mathrm{BCa}$

the same results had been reported in Melanoma LOXIMVI cells in a study by Chang $e t a l^{51}$. Combined with the RIP and biotin pull-down assay results, we calculate that this induction was through the way of specifically binding to the 3 '-UTR of HIF-1A mRNA transcripts. HIF- $1 \alpha$ plays a critical role in regulation of gene transcription as a response to hypoxia, several key regulatory steps participate in the mechanism by which changes in oxygen concentration can be directly transduced into changes in gene expression by HIF- $1 \alpha^{52}$. Under normoxic conditions, HIF- $1 \alpha$ proteins are rapidly ubiquitylated and degraded by the von Hippel-Lindau, and this process depends on the availability of $\mathrm{O}_{2}{ }^{53}$, besides, HIF- $1 \alpha$ transcriptional activity is suppressed under normoxic conditions by hydroxylation of an asparagine residue within its C-terminal transactivation domain, which is mediated by factorinhibiting HIF1 (FIH1) ${ }^{54}$. Our results also showed that CIRBP could increase mRNA stability and protein translation of HIF- $1 \alpha$ in $\mathrm{BCa}$ cell line, this may be part of reason why HIF- $1 \alpha$ could be induced under hypoxia conditions.
HIF-1 $\alpha$ dependent regulations in gene expression usually involve the activation of transcription, especially activate many oncogenes in cancers ${ }^{23,52}$, two publications have reported that PTGIS may be an important target gene of HIF- $1 \alpha^{28,44}$. Our results firstly indicated that HIF$1 \alpha$ could transcriptionally suppress the expression of PTGIS in BCa cells through binding to HRE sequence of the PTGIS promoter region. Further studies demonstrated that PTGIS promoter is hypermethylated in $\mathrm{BCa}$, what's more, overexpression of HIF-1A could increase the expression of PTGIS in SV-HUC-1 cells, which have a low PTGIS gene promoter methylation level. Similarly, after culturing with the demethylating agent, the previous transcriptional suppression would turn to transcriptional activation in $\mathrm{BCa}$ cells. Our research clarified that methylation of PTGIS gene promoter may be an important reason why PTGIS expression is downregulated by HIF- $1 \alpha$ but not upregulated by it in BCa cell line. A few studies have reported the transcriptional suppression of HIF-1 $\alpha$ on target genes promoter activity. A study by Chen et al. showed that HIF- $1 \alpha$ transcriptionally 
suppressed cad-gene expression under hypoxia ${ }^{55}$, another study by Mazure et al. displayed that the existence of a possible competition between HIF- $1 \alpha$ and $\mathrm{c}-\mathrm{Myc}$ that could modulate the transcriptional activity of the AFP gene in response to hypoxia, which resulted in a suppression effect of hypoxia on AFP gene expression ${ }^{56}$. More researches are needed to explore the detailed mechanism for transcriptional suppression of HIF- $1 \alpha$ on PTGIS expression in BCa.

Many studies have reported that PTGIS acted as a tumor suppressor in several cancers ${ }^{30-32}$, our microarray analysis (GEO accession No. GSE76211) revealed that PTGIS is significantly downregulated in BCa tissues, and qRT-PCR analysis results proved this conclusion. In addition, we proved that PTGIS overexpression could suppress the $\mathrm{BCa}$ cells proliferation and migration, together with our demonstration that PTGIS increased the protein level of PPAR- $\gamma$ and suppressed EMT, we demonstrated that PTGIS also acts as a tumor suppressor in BCa. MethHc database showed that PTGIS promotor methylation was negatively correlated with PTGIS mRNA expression levels in $\mathrm{BCa}$, this indicated that DNA methylation is an important reason for obvious downregulation of PTGIS in BCa. In contrast, GEPIA database (http://gepia.cancer-pku.cn/) showed no correlation between HIF-1A and PTGIS expression (Supplementary Figure S1L), indicating that HIF-1A upregulation in $\mathrm{BCa}$ may be a part reason for the downregulation of PTGIS.

Taken together, as shown in mechanism diagram (Fig. 8), our study demonstrated that CIRBP acts as an oncogene during $\mathrm{BCa}$ progression, which could promote $\mathrm{BCa}$ cells proliferation and migration both in vitro and in vivo. Furthermore, CIRBP could increase HIF-1 $\alpha$ expression by binding to the $3^{\prime}$-UTR of HIF-1A mRNA transcripts and increasing its mRNA stability in BCa cell line. In addition, PTGIS is a HIF- $1 \alpha$ target gene, which could suppress $\mathrm{BCa}$ cells proliferation and migration, and overexpression of HIF-1A could suppress the expression of PTGIS in BCa cells by binding to methylated HRE sequence $\left(5^{\prime}\right.$-ccACGTGc-3') of the PTGIS promoter region. what's more, further result suggested that knockdown of CIRBP could promote the expression of PTGIS, meanwhile knockdown of PTGIS could partially rescue siCIRBP induced inhibition of migration and proliferation in BCa cells

\footnotetext{
Acknowledgements

The excellent technical assistance of Yuan Zhu, Shanshan Zhang and Danni Shan is gratefully acknowledged. We thank Dr. Zhongqiang Guo at Departments of Urology, Zhongnan Hospital of Wuhan University, to kindly provide us the p3xFlag-CMV-14-HIF-1A plasmid. This study was supported in part by grants from National Natural Science Foundation of China (grant number 81772730), Hubei Province Health and Family Planning Scientific Research Project (grant number WJ2017H0002) and Natural Science Foundation of Hubei Province of China (grant number 2016CFB113). The
}

funders had no role in study design, data collection and analysis, decision to publish or preparation of the manuscript.

\section{Author details}

'Department of Urology, Zhongnan Hospital of Wuhan University, Wuhan, China. ${ }^{2}$ Department of Biological Repositories, Zhongnan Hospital of Wuhan University, Wuhan, China. ${ }^{3}$ Human Genetics Resource Preservation Center of Hubei Province, Wuhan, China. ${ }^{4}$ Laboratory of Precision Medicine, Zhongnan Hospital of Wuhan University, Wuhan, China. ${ }^{5}$ Department of Urology, LudwigMaximilians-University Munich, Munich, Germany. ${ }^{6}$ Medical Research Institute, Wuhan University, Wuhan, China. ${ }^{7}$ Department of Pathology, Lombardi Comprehensive Cancer Center, Georgetown University Medical School, Washington, DC, USA. ${ }^{8}$ Department of Urology, Massachusetts General Hospital, Harvard Medical School, Boston, MA, USA

\section{Conflict of interest}

The authors declare that they have no conflict of interest.

\section{Publisher's note}

Springer Nature remains neutral with regard to jurisdictional claims in published maps and institutional affiliations.

Supplementary Information accompanies this paper at (https://doi.org/ 10.1038/s41419-018-1109-5).

Received: 11 June 2018 Revised: 14 September 2018 Accepted: 26 September 2018

Published online: 12 October 2018

\section{References}

1. Ferlay, J. et al. Cancer incidence and mortality worldwide: sources, methods and major patterns in GLOBOCAN 2012. Int J. Cancer 136, E359-E386 (2015).

2. Sanli, O. et al. Bladder cancer. Nat. Rev. Dis. Prim. 3, 17022 (2017).

3. Nishiyama, $\mathrm{H}$. et al. A glycine-rich RNA-binding protein mediating coldinducible suppression of mammalian cell growth. J. Cell Biol. 137, 899-908 (1997).

4. Yang, C. \& Carrier, F. The UV-inducible RNA-binding protein A18 (A18 hnRNP) plays a protective role in the genotoxic stress response. J. Biol. Chem. 276, 47277-47284 (2001)

5. Chappell, S. A., Owens, G. C. \& Mauro, V. P. A $5^{\prime}$ leader of Rbm3, a cold stressinduced mRNA, mediates internal initiation of translation with increased efficiency under conditions of mild hypothermia. J. Biol. Chem. 276, 36917-36922 (2001)

6. Wellmann, S. et al. Oxygen-regulated expression of the RNA-binding proteins RBM3 and CIRP by a HIF-1-independent mechanism. J. Cell Sci. 117, 1785-1794 (2004)

7. Artero-Castro, A. et al. Cold-inducible RNA-binding protein bypasses replicative senescence in primary cells through extracellular signal-regulated kinase 1 and 2 activation. Mol. Cell Biol. 29, 1855-1868 (2009).

8. Lleonart, M. E. A new generation of proto-oncogenes: cold-inducible RNA binding proteins. Biochim Biophys. Acta 1805, 43-52 (2010).

9. Liao, Y., Feng, J., Zhang, Y., Tang, L. \& Wu, S. The mechanism of CIRP in inhibition of keratinocytes growth arrest and apoptosis following low dose UVB radiation. Mol. Carcinog. 56, 1554-1569 (2017).

10. Sakurai, T. et al. Cold-inducible RNA-binding protein promotes the development of liver cancer. Cancer Sci. 106, 352-358 (2015).

11. Juan, $Y$. et al. Cold-inducible RNA-binding protein mediates airway inflammation and mucus hypersecretion through a post-transcriptional regulatory mechanism under cold stress. Int J. Biochem Cell Biol. 78, 335-348 (2016).

12. Wang, W., Martindale, J. L., Yang, X., Chrest, F. J. \& Gorospe, M. Increased stability of the p16 mRNA with replicative senescence. EMBO Rep. 6, 158-164 (2005).

13. El-Naggar, A. M. et al. Translational activation of HIF1alpha by YB-1 promotes sarcoma metastasis. Cancer Cell 27, 682-697 (2015).

14. Ivanova, I. G., Park, C. V., Yemm, A. I. \& Kenneth, N. S. PERKVelF2alpha signaling inhibits HIF-induced gene expression during the unfolded protein response 
via YB1-dependent regulation of HIF1alpha translation. Nucleic Acids Res 46, 3878-3890 (2018).

15. Yang, R. et al. Functional significance for a heterogenous ribonucleoprotein A18 signature RNA motif in the 3'-untranslated region of ataxia telangiectasia mutated and Rad3-related (ATR) transcript. J. Biol. Chem. 285, 8887-8893 (2010).

16. Yang, R., Weber, D. J. \& Carrier, F. Post-transcriptional regulation of thioredoxin by the stress inducible heterogenous ribonucleoprotein A18. Nucleic Acids Res 34, 1224-1236 (2006)

17. Zhong, $\mathrm{H}$. et al. Overexpression of hypoxia-inducible factor 1alpha in common human cancers and their metastases. Cancer Res 59, 5830-5835 (1999).

18. Schindl, M. et al. Overexpression of hypoxia-inducible factor 1alpha is associated with an unfavorable prognosis in lymph node-positive breast cancer. Clin. Cancer Res 8, 1831-1837 (2002).

19. Rasheed, S. et al. Hypoxia-inducible factor-1alpha and -2alpha are expressed in most rectal cancers but only hypoxia-inducible factor-1alpha is associated with prognosis. Br. J. Cancer 100, 1666-1673 (2009).

20. Giatromanolaki, A. et al. Relation of hypoxia inducible factor 1 alpha and 2 alpha in operable non-small cell lung cancer to angiogenic/molecular profile of tumours and survival. Br. J. Cancer 85, 881-890 (2001).

21. Theodoropoulos, V. E. et al. Hypoxia-inducible factor 1 alpha expression correlates with angiogenesis and unfavorable prognosis in bladder cancer. Eur. Urol. 46, 200-208 (2004).

22. Forsythe, J. A. et al. Activation of vascular endothelial growth factor gene transcription by hypoxia-inducible factor 1. Mol. Cell Biol. 16, 4604-4613 (1996).

23. Semenza, G. L. Hypoxia-inducible factors: mediators of cancer progression and targets for cancer therapy. Trends Pharmacol. Sci. 33, 207-214 (2012).

24. Dallas, N. A. et al. Functional significance of vascular endothelial growth factor receptors on gastrointestinal cancer cells. Cancer Metastas-. Rev. 26, 433-441 (2007).

25. Mak, P. et al. ERbeta impedes prostate cancer EMT by destabilizing HIF-1alpha and inhibiting VEGF-mediated snail nuclear localization: implications for Gleason grading. Cancer Cell 17, 319-332 (2010).

26. Krishnamachary, B. et al. Hypoxia-inducible factor-1-dependent repression of E-cadherin in von Hippel-Lindau tumor suppressor-null renal cell carcinoma mediated by TCF3, ZFHX1A, and ZFHX1B. Cancer Res 66, 2725-2731 (2006).

27. Semenza, G. L. Oxygen sensing, hypoxia-inducible factors, and disease pathophysiology. Annu Rev. Pathol. 9, 47-71 (2014).

28. Manalo, D. J. et al. Transcriptional regulation of vascular endothelial cell responses to hypoxia by HIF-1. Blood 105, 659-669 (2005).

29. Spisni, E. et al. Colocalization prostacyclin (PG/2) synthase--caveolin-1 in endothelial cells and new roles for PGI2 in angiogenesis. Exp. Cell Res 266 31-43 (2001).

30. Keith, R. L. et al. Manipulation of pulmonary prostacyclin synthase expression prevents murine lung cancer. Cancer Res 62, 734-740 (2002).

31. Gupta, R. A. et al. Prostacyclin-mediated activation of peroxisome proliferatoractivated receptor delta in colorectal cancer. Proc. Natl. Acad. Sci. USA 97, 13275-13280 (2000)

32. Cathcart, M. C., Reynolds, J. V., O'Byrne, K. J. \& Pidgeon, G. P. The role of prostacyclin synthase and thromboxane synthase signaling in the development and progression of cancer. Biochim Biophys. Acta 1805, 153-166 (2010).

33. Kitamoto, S. et al. Expression of MUC17 is regulated by HIF1alpha-mediated hypoxic responses and requires a methylation-free hypoxia responsible element in pancreatic cancer. PLOS ONE 7, e44108 (2012).

34. Horiuchi, A. et al. Hypoxia upregulates ovarian cancer invasiveness via the binding of HIF-1alpha to a hypoxia-induced, methylation-free hypoxia response element of S100A4 gene. Int J. Cancer 131, 1755-1767 (2012).

35. Rossler, J. et al. Hypoxia-induced erythropoietin expression in human neuroblastoma requires a methylation free HIF-1 binding site. J. Cell Biochem 93 153-161 (2004).
36. Cebola, I. et al. Epigenetics override pro-inflammatory PTGS transcriptomic signature towards selective hyperactivation of PGE2 in colorectal cancer. Clin. Epigenetics 7, 74 (2015).

37. Frigola, J. et al. Hypermethylation of the prostacyclin synthase (PTGIS) promoter is a frequent event in colorectal cancer and associated with aneuploidy. Oncogene 24, 7320-7326 (2005)

38. Torii, S., Yamamoto, T., Tsuchiya, Y. \& Nishida, E. ERK MAP kinase in G cell cycle progression and cancer. Cancer Sci. 97, 697-702 (2006).

39. Dhillon, A. S., Hagan, S., Rath, O. \& Kolch, W. MAP kinase signalling pathways in cancer. Oncogene 26, 3279-3290 (2007).

40. Wang, G. et al. Simvastatin induces cell cycle arrest and inhibits proliferation of bladder cancer cells via PPARgamma signalling pathway. Sci. Rep. 6, 35783 (2016).

41. Cao, R. et al. Decreased TRPM7 inhibits activities and induces apoptosis of bladder cancer cells via ERK1/2 pathway. Oncotarget 7, 72941-72960 (2016).

42. Jian, F. et al. Cold inducible RNA binding protein upregulation in pituitary corticotroph adenoma induces corticotroph cell proliferation via Erk signaling pathway. Oncotarget 7, 9175-9187 (2016).

43. Lee, H. N., Ahn, S. M. \& Jang, H. H. Cold-inducible RNA-binding protein promotes epithelial-mesenchymal transition by activating ERK and p38 pathways. Biochem Biophys. Res Commun. 477, 1038-1044 (2016).

44. Mimura, I. et al. Dynamic change of chromatin conformation in response to hypoxia enhances the expression of GLUT3 (SLC2A3) by cooperative interaction of hypoxia-inducible factor 1 and KDM3A. Mol. Cell Biol. 32, 3018-3032 (2012).

45. Masoud, G. N. \& Li, W. HIF-1alpha pathway: role, regulation and intervention for cancer therapy. Acta Pharm. Sin. B 5, 378-389 (2015).

46. Tate, P. H. \& Bird, A. P. Effects of DNA methylation on DNA-binding proteins and gene expression. Curr. Opin. Genet Dev. 3, 226-231 (1993).

47. Okami, J., Simeone, D. M. \& Logsdon, C. D. Silencing of the hypoxia-inducible cell death protein BNIP3 in pancreatic cancer. Cancer Res 64, 5338-5346 (2004).

48. Sakurai, T. et al. Stress response protein cirp links inflammation and tumorigenesis in colitis-associated cancer. Cancer Res 74, 6119-6128 (2014).

49. Meloche, S. \& Pouyssegur, J. The ERK1/2 mitogen-activated protein kinase pathway as a master regulator of the G1- to S-phase transition. Oncogene $\mathbf{2 6}$ 3227-3239 (2007).

50. Kim, M. Y., Hur, J. \& Jeong, S. Emerging roles of RNA and RNA-binding protein network in cancer cells. BMB Rep. 42, 125-130 (2009).

51. Chang, E. T., Parekh, P. R., Yang, Q., Nguyen, D. M. \& Carrier, F. Heterogenous ribonucleoprotein A18 (hnRNP A18) promotes tumor growth by increasing protein translation of selected transcripts in cancer cells. Oncotarget $\mathbf{7}$, 10578-10593 (2016)

52. Lendahl, U., Lee, K. L., Yang, H. \& Poellinger, L. Generating specificity and diversity in the transcriptional response to hypoxia. Nat. Rev. Genet 10, 821-832 (2009)

53. Mahon, P. C., Hirota, K. \& Semenza, G. L. FIH-1: a novel protein that interacts with HIF-1alpha and VHL to mediate repression of HIF-1 transcriptional activity. Genes Dev. 15, 2675-2686 (2001).

54. Lando, D. et al. FIH-1 is an asparaginyl hydroxylase enzyme that regulates the transcriptional activity of hypoxia-inducible factor. Genes Dev. 16, 1466-1471 (2002).

55. Chen, K. F., Lai, Y. Y., Sun, H. S. \& Tsai, S. J. Transcriptional repression of human cad gene by hypoxia inducible factor-1alpha. Nucleic Acids Res 33, 5190-5198 (2005).

56. Mazure, N. M. et al. Repression of alpha-fetoprotein gene expression under hypoxic conditions in human hepatoma cells: characterization of a negative hypoxia response element that mediates opposite effects of hypoxia inducible factor-1 and c-Myc. Cancer Res 62, 1158-1165 (2002). 